\title{
The ice and Potts models in
}

\section{statistical mechanics}

\section{Stewart Beaton Kelland}

This thesis deals with two two-dimensional lattice models in statistical mechanics. The first of these is the zero-field 20-vertex model on a triangular lattice. This model is the analogue to the ice-type, zero-field six-vertex model on a square lattice. We solve this problem exactly for a restricted set of vertex weights. The other model is the $q$-component Potts model on a square lattice. This model is equivalent to a staggered ice-type model on a square lattice. Using a variational method we estimate the critical exponent $\beta$ for several values of $q$.

In Part I we outline the fundamental statistical mechanical ideas needed, including the definition of the partition function and its relation to the thermodynamic quantities. Mention is also made of the existence and uniqueness of the thermodynamic limit for these models.

The 20-vertex model is discussed in Part II. The transfer matrix approach is adopted and a Bethe ansatz is used for the eigenvector. Unlike the square lattice ice-type model, the triangular lattice 20-vertex model is only soluble by this method for a restricted set of vertex weights. In particular, the ten parameters determining the vertex weights must be expressed in terms of four independent parameters. 'This results in only' one type of non-trivial temperature independent solution. This solution exhibits critical behaviour similar to the square lattice KDP model. The general solution has three states: disordered, antiferroelectric, and ferroelectric. Which state the model is in is determined by one of the above four parameters. The transition from the disordered to the anti-

Received. 8 June 1976. Thesis submitted to the Australian National University, February 1976. Degree approved, June 1976. Supervisor: Dr R. J. Baxter. 
ferroelectric state is of infinite-order, and from the disordered to the ferroelectric state is of finite-order. We also compare our results to the free-fermion solution of the same model and note where the two methods overlap.

In Part III the Potts model is considered. The model is defined and shown to be equivalent to a staggered ice-type model. A variational principle for the eigenvalues of the transfer matrix of the staggered ice model is determined. Using the zero-temperature solution as a guide a simple form for a sequence of approximations to the eigenvector, below $T_{c}$, is developed. By using the variational principle we arrive at a set of matrix equations determining the eigenvector at a given level of approximation. By noting some of the symmetries of the transfer matrix we are able to separate some of the dependences of the matrix elements and consequently simplify the original set of equations. We are also able to express the percolation probability (the analogue of the spontaneous magnetization for general values of $q$ ) of the Potts model as a simple function of these matrix elements. At each level of approximation there is a set of algebraic equations to solve. We have used an electronic computer to solve these equations for the first five levels of approximation, and for several values of $q$ between zero and four. With our results we estimate the critical exponent $\beta$ for the Potts model. These estimates agree reasonably well with previous work and indicate $\beta=0.145 \pm .017$ for $q=1$. 